12

\title{
Синтез электродных конфигураций, сохраняющих для краевых электрических полей свойство однородности по Эйлеру
}

\author{
(C) Ю.К. Голиков, ${ }^{1}$ А.С. Бердников, ${ }^{2, \uparrow}$ А.С. Антонов, ${ }^{2,3}$ Н.К. Краснова, ${ }^{1}$ К.В. Соловьёв ${ }^{1}$ \\ ${ }^{1}$ Санкт-Петербургский политехнический университет Петра Великого, \\ 195251 Санкт-Петербург, Россия \\ ${ }^{2}$ Институт аналитического приборостроения РАН, \\ 190103 Санкт-Петербург, Россия \\ ${ }^{3}$ Физико-технический институт им. А.Ф. Иоффре РАН, \\ 194021 Санкт-Петербург, Россия \\ "e-mail: asberd@yandex.ru
}

(Поступило в Редакцию 26 августа 2017 г.)

\begin{abstract}
Электрические поля, идеально однородные по Эйлеру, являются полезным инструментом для синтеза электронно- и ионно-оптических систем с уникальными свойствами. Однако реальные краевые поля имеют свойство разрушать идеальность теоретически придуманных схем. Рассмотрен способ синтеза краевых полей, который сохраняет их однородность по Эйлеру и, следовательно, сохраняет теоретически предсказываемые оптимальные характеристики электронно- и ионно-оптических систем такого типа.
\end{abstract}

DOI: 10.21883/JTF.2018.04.45732.2483

Электрическими полями, однородными по Эйлеру с порядком однородности $n$, называются поля, у которых напряженность электрического поля $\mathbf{E}(x, y, z)$ удовлетворяет условию $\mathbf{E}(\lambda x, \lambda y, \lambda z)=\lambda^{n-1} \mathbf{E}(x, y, z)$ при любых $\lambda>0$. Движение заряженных частиц в таких полях подчиняется принципу подобия траекторий $[1,2]$, поэтому использование электрических полей указанного вида гарантирует хорошие характеристики для электронных энергоспектрографов [3-5], масс-спектрографов [6,7], поворотных и призматических систем $[8,9]$.

Однородные по Эйлеру потенциалы, заданные в аналитическом виде, позволяют конструировать весьма сложные оптические схемы с уникальными полезными свойствами [3-9]. Однако на этом пути часто возникает необходимость ввода и вывода частиц „сквозь“ аналитически рассчитанные электроды. Паразитные краевые поля, возникающие для электродных конфигураций с дополнительными диафрагмами, уже не обладают свойством однородности по Эйлеру и поэтому разрушают для реального устройства его идеальные свойства. Это ставит под сомнение саму идею практической полезности синтеза оптических систем, использующих принцип однородности по Эйлеру.

В настоящей работе решается задача создания таких электродных конфигураций, где краевые поля с самого начала будут обладать свойством однородности по Эйлеру. Хотя оптимальный режим работы подобных устройств будет достигаться уже при других параметрах, чем у исходной „идеальной“ схемы, однако теоретически предсказываемые оптические характеристики, которые следуют из свойства однородности по Эйлеру, будут сохранены.

Рассмотрим электростатическое зеркало [10] с полем, которое является однородным по Эйлеру и задается электрическим потенциалом нулевого порядка

$$
U(x, y, z)= \begin{cases}U_{0}\left(\operatorname{arctg} \frac{y}{x}-\frac{y}{x+\sqrt{x^{2}+y^{2}+z^{2}}}\right) & \text { при } y \geq 0, \\ 0 & \text { при } y \leq 0 .\end{cases}
$$

В соответствии с принципом подобия траекторий $[1,2,8,9]$ это поле преобразует параллельный монохроматический пучок на входе в параллельный же монохроматический пучок на выходе, причем угол поворота и выходные характеристики пучка будут существенным образом зависеть от входной энергии.

На рис. 1, $а$ показаны криволинейные электроды, создающие такое электрическое поле, на рис. $1, b$ - параллельные траектории заряженных частиц при разных начальных энергиях. Такая система, в которой угол поворота параллельного пучка зависит от энергии, может использоваться как поворотная система, монохроматор, энергоанализатор с дисперсией по углу.

Недостатком системы на рис. 1 (кроме криволинейного электрода сложной формы) является ввод и вывод заряженных частиц сквозь заземленный сплошной электрод в виде вертикальной плоскости $y=0$. Тонкая сетка на входе является плохим конструктивным решением, а бессеточная диафрагма общего вида порождает краевые поля, не являющиеся однородными по Эйлеру и разрушающие свойство поля (1) преобразовывать параллельные траектории на входе в параллельные траектории на выходе.

Можно ли сконструировать для потенциала (1) бессеточную диафрагму с таким краевым полем, которое оставит в целости базовое свойство поворотной системы преобразовывать параллельные траектории на входе в параллельные траектории на выходе? Ответ на этот вопрос положительный. Рассмотрим в общем виде задачу 


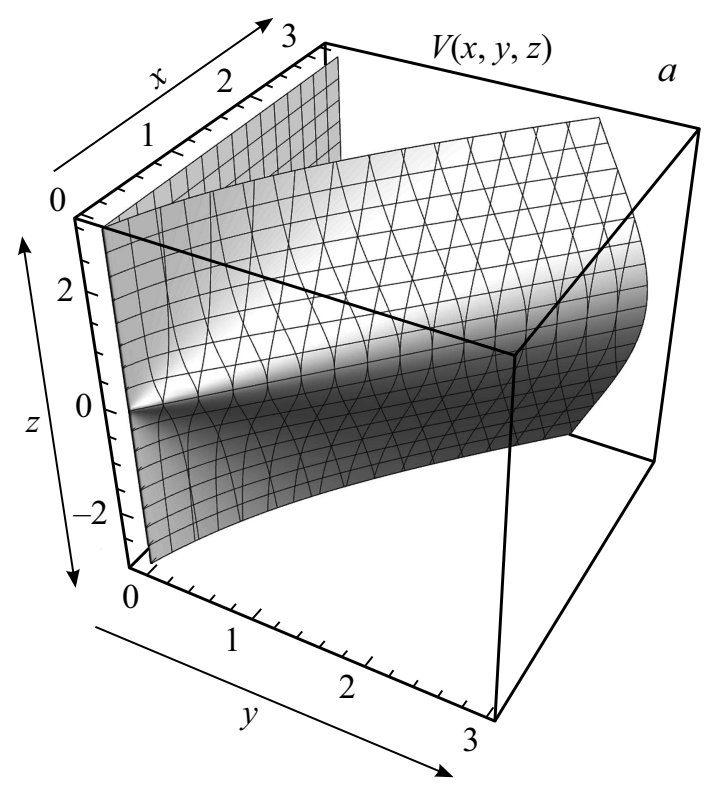

$b$

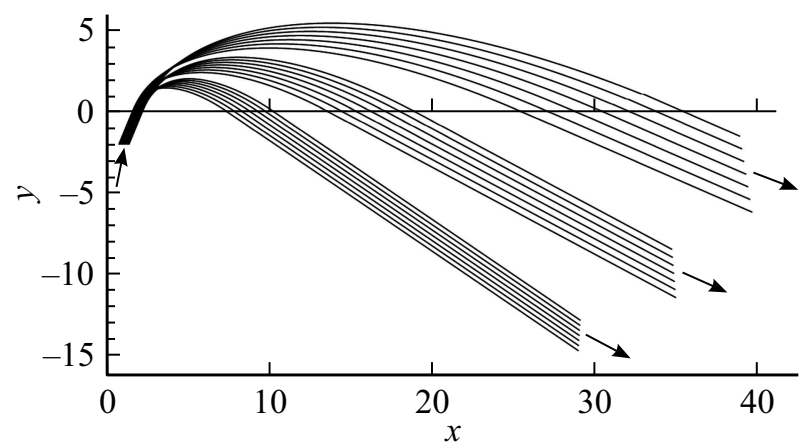

Рис. 1. Электростатическое зеркало с однородным по Эйлеру электрическим потенциалом, заданным формулой (1): $a-$ трехмерные электроды, $b$ - параллельные монохроматические пучки траекторий в плоскости симметрии.
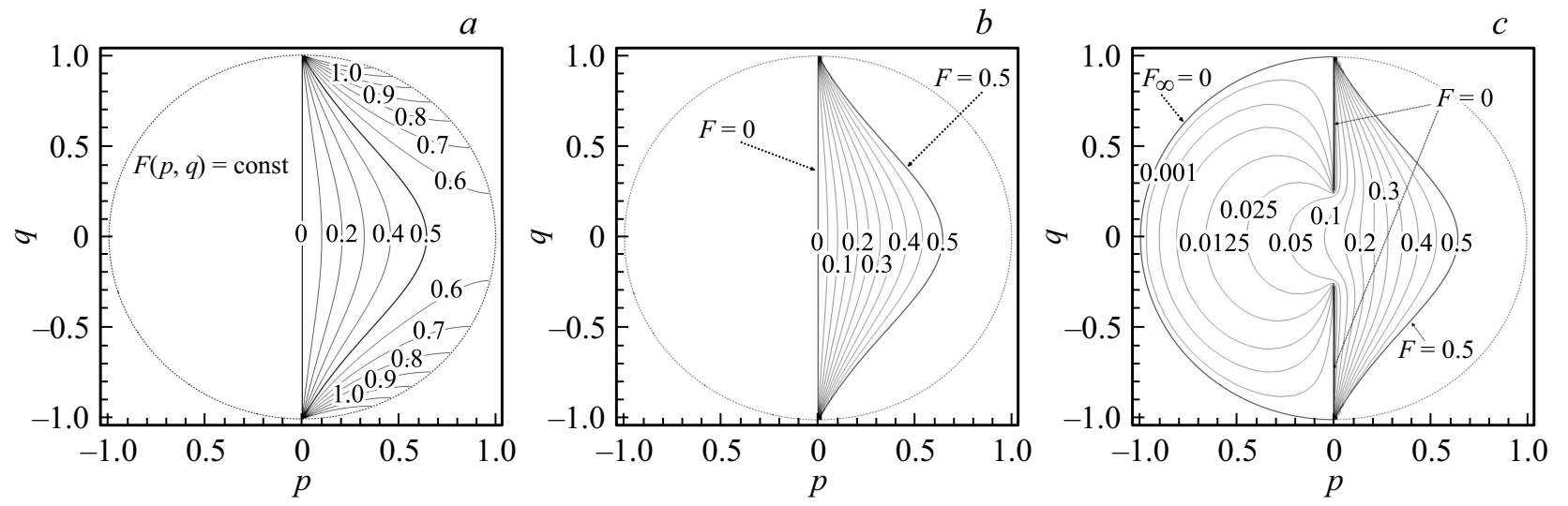

Рис. 2. Отображение на плоскость $(p, q)$ трехмерных электродов зеркала, показанного на рис. 1 , и его эквипотенциальные линии: $a$ - эквипотенциальные линии исходного потенциала, $b$ - краевая задача для сплошного экрана и эквипотенциальные линии точного аналитического решения, $c$ - краевая задача для экрана с „дыркой“ и эквипотенциальные линии численного решения. Окружность $p^{2}+q^{2}=1$, обозначенная пунктиром, образ бесконечно удаленных точек $x, y, z$.

синтеза однородного по Эйлеру краевого поля. Пусть $U(x, y, z)$ - однородный по Эйлеру электрический потенциал порядка $n$. Так как $U-$ однородная функция, ее можно представить как $U=r^{n} F(p, q)([11,12])$, где $r=\sqrt{x^{2}+y^{2}+z^{2}}, p=y /(x+r), q=z /(x \mid r), F-$ новая функция. Функция $U$ удовлетворяет трехмерному уравнению Лапласа, поэтому функция $F$ удовлетворяет двумерному эллиптическому уравнению в частных производных

$$
\partial^{2} F / \partial p^{2}+\partial^{2} F / \partial q^{2}+F\left[4 n(n+1) /\left(1+p^{2}+q^{2}\right)^{2}\right]=0 .
$$

На рис. 2, $а$ показаны эквипотенциальные линии для функции $F$, соответствующей потенциалу (1), нарисованные в координатах $(p, q)$. Жирными линиями выде- лены эквипотенциальные линии, соответствующие электродам на рис. $1, a$.

Если теперь для области, ограниченной жирными линиями, поставить и решить краевую задачу для уравнения (2) (рис. 2, $b$ ), то очевидным образом ответом будет исходный потенциал (1), пересчитанный в координаты $(r, p, q)$. Но если в выходном электроде прорезать ,дырку“, как это сделано на рис. 2, $c$, и решить (вероятно, численно) краевую задачу уже для этой области, то ответом будет совсем другая функция $\hat{F}$ (рис. 2,c). Теперь после перехода от функции $\hat{F}$ к функции $\hat{U}$ по формуле $\hat{U}=r^{n} \hat{F}(p, q)$ получится новый трехмерный потенциал, гармонический и однородный по Эйлеру, но с „дыркой“ сложной формы на месте сплошного электрода $y=0$. 

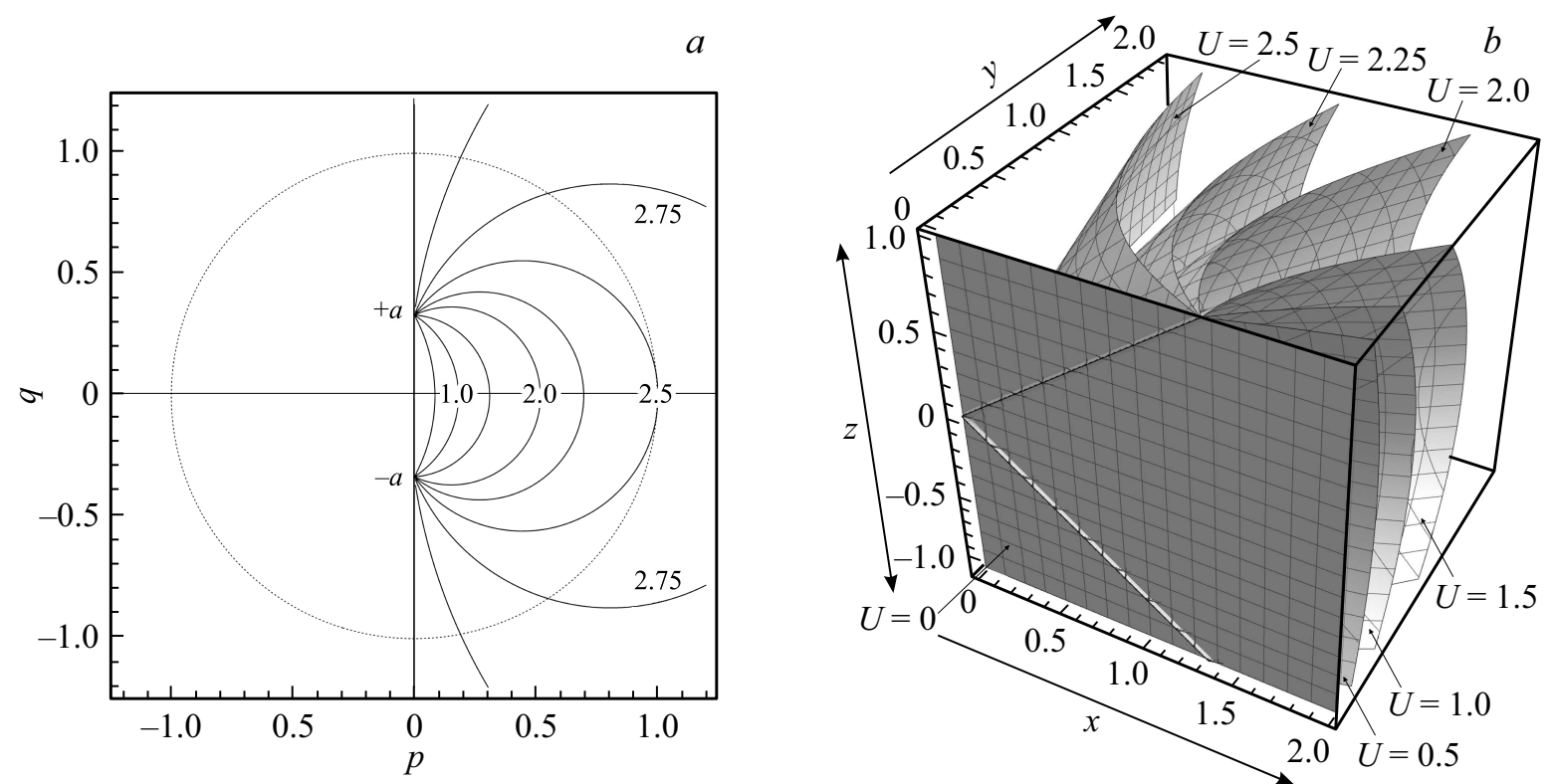

Рис. 3. Эквипотенциальные линии в плоскости $(p, q): a-$ эквипотенциальные поверхности в трехмерном пространстве, $b-$ для электрического потенциала, заданного формулой (4).

Легко убедиться, что для рассмотренного примера синтезированная „дырка“ будет иметь вид клиновидной щели, проделанной в исходной плоскости $y=0$. Такая поворотная система в полной мере сохраняет параллельность траекторий, но по сравнению с исходной системой угол влета, угол поворота, угловая хроматическая дисперсия и уширение пучка слегка изменятся в зависимости от угла клиновидной щели. Кроме того, если размер клиновидной щели сопоставим с расстоянием до внутреннего электрода, то определенному искажению нужно будет подвергнуть и внутренний криволинейный электрод, чтобы сохранить для полученного поля свойство однородности по Эйлеру. Однако если клиновидная щель, сделанная в плоскости $y=0$, будет достаточно узкой, то искажения формы внутреннего криволинейного электрода будут незначительными и ими можно пренебречь.

В конечном итоге предлагаемый метод синтеза бессеточного краевого поля, сохраняющего свойство однородности по Эйлеру, состоит из следующих шагов: a) для исходного однородного скалярного потенциала строятся идеальные электроды как точные эквипотенциальные поверхности; б) при замене переменных $(x, y, z) \rightarrow(r, p, q)$ эти границы отображаются на плоскость $(p, q)$, а краевая задача для трехмерного уравнения Лапласа - на краевую задачу для уравнения (2) с заданными границами, являющимися образами электродов; в) исходная конфигурация границ искажается, чтобы создать в нужном месте бессеточный вход и/или выход; г) для искаженной границы решается новая краевая задача для уравнения (2); д) полученное решение преобразуется в новый трехмерный потенциал, который можно рассматривать как возмущение исходного потен- циала; е) эквипотенциальные поверхности для нового потенциала обеспечат форму бессеточных полезадающих элементов, сохраняющих однородность поля. Тем самым для однородного по Эйлеру идеального поля будет синтезирован такой бессеточный вариант краевого поля, у которого в полной мере сохранится свойство однородности по Эйлеру. Эффективнее, но и сложнее использовать при конструировании краевой задачи асимптотическое распределение функции $F$ на „бесконечно удаленной“ окружности $p^{2}+q^{2}=1$ (рис. $\left.2, a\right)-$ тогда эквипотенциальные поверхности для нового однородного потенциала $U$ будут определены во всем пространстве.

Когда электрод с привнесенной „дыркой“ имеет нулевой потенциал, клиновидная „дырка“ в исходном электроде будет типичным ответом для задачи синтеза краевого поля, однородного по Эйлеру. Если условие на малость размера щели по сравнению с расстояниями между электродами соблюдено, то нет необходимости выполнять описанную выше процедуру вычисления точного профиля электрода с бессеточной диафрагмой, можно сразу переходить к исследованию оптических свойств новой системы при наличии клиновидной щели, имеющей нужный размер. Однако это утверждение справедливо, только если речь идет об электродах, для которых однородный потенциал обращается в нуль: электроды с ненулевыми потенциалами после добавления искусственной „дырки“ искажаются весьма значительно.

Следуя этой схеме рассуждений, в работе [6] рассчитывается магнитный экран с клиновидной щелью, который обеспечивает однородное краевое поле для постоянного магнита с полюсами сложной формы, создающими магнитное поле с порядком однородности $n=3 / 2$. Идей- 
но близкий подход, но с более сложными уравнениями для функции $F$, рассматривается в работе [13].

В качестве примера рассмотрим отклоняющую систему, состоящую из двух электродов в виде отражающего кругового конического сегмента и заземленной плоскости. Однородный электрический потенциал нулевого порядка для такой системы записывается в аналитической форме в переменных $(p, q)$ как

$$
\begin{aligned}
U(p, q) & =U_{0}\left(\operatorname{arctg}\left(\frac{p}{a+q}\right)+\operatorname{arctg}\left(\frac{p}{a-q}\right)\right) \\
& =U_{0} \operatorname{arctg}\left(\frac{2 a p}{a^{2}-\left(p^{2}+q^{2}\right)}\right),
\end{aligned}
$$

а в переменных $x, y, z$ как

$$
\begin{aligned}
& U(x, y, z)= \\
& = \begin{cases}U_{0}\left(\operatorname{arctg} \frac{y}{a x-\left(\frac{1-a^{2}}{2 a}\right) \frac{y^{2}+z^{2}}{x+\sqrt{x^{2}+y^{2}+z^{2}}}}\right) & \text { при } y \geq 0, \\
0 & \text { при } y \leq 0,\end{cases}
\end{aligned}
$$

где параметр $U_{0}$ характеризует разность потенциалов между электродами, а безразмерный параметр $a$, принадлежащий промежутку $0<a<1$, управляет геометрической формой электродов.

Эквипотенциальные линии для функции (3) (рис. 3, a) представляют собой симметричные дуги окружностей с центром на линии $q=0$, которые соединяют точки $(0,+a)$ и $(0,-a)$ и подчиняются уравнениям

$$
(p+a \lambda)^{2}+q^{2}=a^{2}\left(1+\lambda^{2}\right),
$$

где $\lambda$ - безразмерный параметр, характеризующий рассматриваемую эквипотенциальную линию. Дополнительной эквипотенциальной линией для функции (3) будет служить прямая линия $p=0$. В результате эквипотенциальными поверхностями для функции (4) (рис. $3, b)$ служат плоскость $y=0$ с нулевым потенциалом и сегменты круговых конусов с вершинами в начале координат, которые удовлетворяют алгебраическим уравнениям

$$
\begin{aligned}
-4 a^{2} x^{2} & +4 a\left(1+a^{2}\right) \lambda x y \\
& +\left(\left(1-a^{2}\right)^{2}-4 a^{2} \lambda^{2}\right) y^{2}+\left(1-a^{2}\right)^{2} z^{2}=0
\end{aligned}
$$

и могут быть записаны в параметрическом виде как

$$
\begin{gathered}
x(\tau, \varphi)=\tau \cos \varepsilon+s \tau \cos \varphi \sin \varepsilon, \\
y(\tau, \varphi)=-\tau \sin \varepsilon+s \tau \cos \varphi \cos \varepsilon, \\
z(\tau, \varphi)=s \tau \sin \varphi,
\end{gathered}
$$

где $\varepsilon=\operatorname{arctg}\left(2 a \lambda /\left(1+a^{2}\right)\right)-$ наклон оси конуса, лежащей в плоскости $O X Y$, относительно координатной оси $O X, s=2 a \sqrt{1+\lambda^{2}} /\left(1-a^{2}\right)$ - тангенс угла раствора конуса, $\tau \in(0,+\infty)$ и $\varphi \in(-\pi,+\pi)$ - параметры, при варьировании которых точка пробегает всю коническую эквипотенциальную поверхность.
Зависимость угла вылета пучка от энергии $\varepsilon$ и угла раствора клиновидной бессеточной диафрагмы $\beta$ в полуконической поворотной системе для угла влета пучка, равного $55^{\circ}$

\begin{tabular}{c|c|c|c|c}
\hline \multirow{2}{*}{$\varepsilon / V_{0}$} & \multicolumn{4}{|c}{$\operatorname{tg}(\beta)$} \\
\cline { 2 - 5 } & 0 & 0.12 & 0.15 & 0.18 \\
\hline 0.5 & $-49.42^{\circ}$ & $-52.67^{\circ}$ & $-53.94^{\circ}$ & $-55.26^{\circ}$ \\
0.9 & $-45.30^{\circ}$ & $-47.33^{\circ}$ & $-48.16^{\circ}$ & $-49.04^{\circ}$ \\
1.3 & $-41.38^{\circ}$ & $-42.88^{\circ}$ & $-43.50^{\circ}$ & $-44.16^{\circ}$
\end{tabular}

Эквипотенциальные конусы пересекаются друг с другом и с плоскостью $y=0$ вдоль общих прямых линий $z= \pm k x$, где $k=2 a /\left(1-a^{2}\right)$. Эти прямые состоят из сингулярных точек электрического поля. Поскольку указанные точки расположены вдалеке от области, в которой происходит интересующее нас движение заряженных частиц, то искажение реальных электродов в этой области пространства с целью устранения сингулярностей на оптических характеристиках рассматриваемой системы не сказывается. Следует также отметить, что только те участки эквипотенциальных линий на плоскости $(p, q)$, которые содержатся внутри окружности $p^{2}+q^{2}=1$ (образ бесконечно удаленных точек трехмерного пространства), имеют своим прототипом эквипотенциальные поверхности реального электрического поля. Например, поверхности (6) имеют смысл только для значений $-\left(1-a^{2}\right) / 2 a<\lambda<+\infty$.

В качестве первого (входного) электрода удобно выбрать вертикальную плоскость $y=0$, к которой будет прикладываться нулевой потенциал. С точки зрения электрического поля все эквипотенциальные конические поверхности, когда их используют как отражающий электрод, будут равноценны, если только траектории не углубляются внутрь поворотного устройства слишком далеко. Поэтому в роли отражающего электрода удобно использовать круговой конус (точнее, половину кругового конуса, ограниченную плоскостью $y=0)$ с вершиной в начале координат, варьируемым тангенсом угла раствора конуса, обозначаемым как $k$, и главной осью, расположенной вдоль координатной оси $O X$ (это соответствует выбору $\lambda=0$ в формулах (6),(7)). Чтобы выражение (4) соответствовало электрическому полю, создаваемому с помощью такой системы электродов, параметр $U_{0}$ должен вычисляться как $U_{0}=(2 / \pi) V_{0}$, где $V_{0}-$ потенциал на выбранном отражающем электроде, имеющего форму кругового полуконуса с главной осью в виде оси $O X$ и соответствующим углом раствора. Безразмерный параметр $a$, подставляемый в формулы (3), (4), должен вычисляться как $a=k /\left(1+\sqrt{1+k^{2}}\right)$.

Проверим на численном примере, насколько высказанные выше теоретические ожидания выполняются в действительности. В качестве тестовой системы рассмотрим электрическое поле с потенциалом (4), где вместо сплошной заземленной плоскости $y=0$ име- 

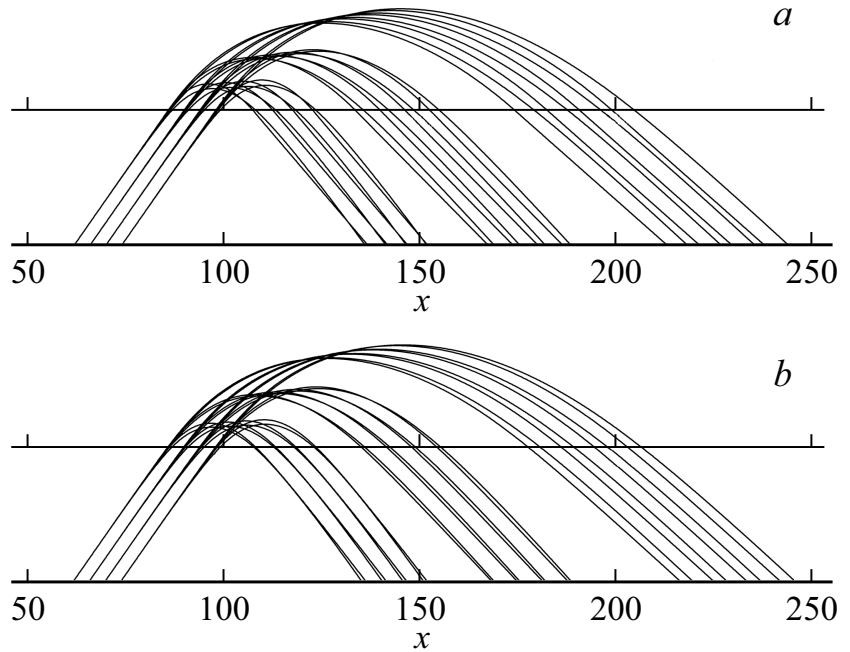

Рис. 4. Результаты отклонения параллельных пучков заряженных частиц с тремя разными фиксированными энергиями, рассчитанными в программе SIMION и совмещенными на одном рисунке для бессеточных клиновидных диафрагм с двумя разными углами раствора, $\operatorname{tg}(\beta): a-0$ и $0.15, b-0.12$ и 0.18 . По оси $x$ отложено количество ячеек, 0 соответствует вершине конуса.

ется бессеточная диафрагма, обеспечивающая свободный ввод и вывод заряженных частиц. Вместо того чтобы аккуратно восстанавливать по описанной выше методике возмущенное краевое поле (и, следовательно, точную форму заземленной бессеточной диафрагмы и отражающего электрода, которые бы обеспечивали для электрического поля однородность по Эйлеру), сразу рассмотрим эффект от введения клиновидной бессеточной диафрагмы для заземленной плоскости $y=0$ при сохранении отражающего полуконического электрода в неизменном виде.

На рис. 4 показаны траектории заряженных частиц, рассчитанные с помощью программы SIMION [14], для „идеального“ случая с бесконечно мелкой сеткой на месте плоскости $y=0$ и для нескольких растворов клиновидной бессеточной диафрагмы. Наглядно видно, что в соответствии с теоретическими ожиданиями угол поворота и другие оптические характеристики выходного пучка весьма ощутимо меняются при варьировании размеров диафрагмы (см. таблицу). Несмотря на это, возмущенная поворотная система по-прежнему переводит входной параллельный монохроматический пучок заряженных частиц в выходной параллельный монохроматический пучок заряженных частиц.

В данном примере угол раствора конуса $\alpha$ был выбран таким, что $\operatorname{tg}(\alpha)=0.6$, а углы раствора клиновидной бессеточной диафрагмы $\beta$ такими, что $\operatorname{tg}(\beta)=0$ (случай идеальной сетки вместо бессеточной диафрагмы), $\operatorname{tg}(\beta)=0.12,0.15$ и 0.18. Были определены траектории заряженных частиц в плоскости симметрии $z=0$, составляющих параллельные монохроматические пучки с энергиями 0.5, 0.9 и 1.3 (в единицах потенциала на отражающем электроде). Объем области моделирования составлял $500 \times 1000 \times 500$ ячеек, что в совокупности с методикой сглаживания поверхностей электродов позволило получить приемлемую точность численного расчета траекторий заряженных частиц. В частности, отклонение траекторий от параллельности в каждом из монохроматических пучков не превышало $0.001^{\circ} \mathrm{C}$.

\section{Список литературы}

[1] Голиков Ю.К., Краснова Н.К. // Прикладная физика. 2007. № 2. C. 5-11.

[2] Аверин И.А., Бердников А.С., Галль Н.Р. // Письма в ЖТФ. 2017. Т. 43. Вып. 3. С. 39-43.

[3] Голиков Ю.К., Краснова Н.К. Теория синтеза электростатических энергоанализаторов. СПб.: Изд-во Политехн. унта. 2010. 409 c.

[4] Голиков Ю.К., Краснова Н.К., Абрамёнок О.А. // Прикладная физика. 2011. № 5. С. 69-73.

[5] Краснова Н. К. // ЖТФ. 2012. Т. 82. Вып. 8. С. 105-109.

[6] Бердников А.С., Аверин И.А., Голиков Ю.К. // Массспектрометрия. 2015. Т. 12. № 4. С. 272-281.

[7] Бердников А.С., Аверин И.А., Голиков Ю.К. // Массспектрометрия. 2016. Т. 13. № 1. С. 11-20.

[8] Габдуллин П.Г., Голиков Ю.К., Краснова Н.К., Давыдов С.Н. // ЖТФ. 2000. Т. 70. Вып. 2. С. 91-94.

[9] Габдуллин П.Г., Голиков Ю.К., Краснова Н.К., Давыдов С.Н. // ЖТФ. 2000. Т. 70. Вып. 3. С. 44-47.

[10] Бердников А.С., Краснова Н.К. // Научное приборостроение. 2015. Т. 25. № 2. С. 69-90.

[11] Смирнов В.И. Курс высшей математики. Т. 1. М.: Наука, 1974. $480 \mathrm{c}$.

[12] Фихтенгольи Г.М. Курс дифференциального и интегрального исчисления. Т. 1. М.: Физматлит, 2001. 616 с.

[13] Аверин И. А., Бердников А. С. // Успехи прикладной физики. 2016. Т. 4. № 1. С. 5-8.

[14] Электронный ресурс. Программа SIMION. URL: режим доступа http://simion.com/. 